\title{
Development of a Versatile Robotic System with Multiple Training Modes for Upper-Limb Rehabilitation Study
}

Furui Wang $^{1 *}$, Duygun Erol Barkana ${ }^{2}$ and Nilanjan Sarkar ${ }^{3}$

${ }^{1}$ Abbott Point of Care, Abbott Laboratories, Princeton, NJ, 08540, USA

${ }^{2}$ Department of Electrical and Electronics Engineering, Yeditepe University, Istanbul, Turkey

${ }^{3}$ Department of Mechanical Engineering, Vanderbilt University, Nashville, TN, 37212, USA

\begin{abstract}
This paper presents the development of a versatile robotic system with multiple training modes to serve as a test-bed that will facilitate the study of upper-limb rehabilitation following stroke. Seven different training modes, i.e., passive, low-impedance, assist-as-needed, resist-as-needed, visual error augmentation, viscous force field and force perturbation, have been integrated in this robotic system. A hierarchic control system is developed to coordinate these training modes. Initial experiments on unimpaired participants have verified that the robotic system is able to provide the above training modes properly.
\end{abstract}

Keywords: Rehabilitation Robotics; Multiple Training Modes; Upper-limb movement; Motor learning and adaptation

\section{Introduction}

Stroke is a highly prevalent condition [1], especially among the elderly, that results in high costs to the individual and society [2]. According to the American Heart Association (2013), in the U.S., approximately 795000 people suffer a first or recurrent stroke each year [1]. Of these, $60-75 \%$ will live beyond one year after incidence, resulting in a current stroke population of 6.5 million $[1,3,4]$. Arm function is acutely impaired in a large majority of those diagnosed with stroke [5]. It is a leading cause of disability, commonly involving deficits of motor function. Recent clinical results have indicated that movement assisted therapy can have a significant beneficial impact on a large segment of the population affected by stroke or other motor deficit disorders. In the last few years, robot-assisted rehabilitation for the stroke patients has been an active research area, which provides repetitive movement exercise and standardized delivery of therapy with the potential of enhancing quantification of the therapeutic process [6-13].

Various robot-assisted rehabilitation systems are developed for the upper-limb rehabilitation such as MIT-MANUS [6,7], Assisted Rehabilitation and Measurement (ARM) Guide [8,9]. Mirror Image Movement Enabler (MIME) [10-12] and GENTLE/s [13]. Studies with these robotic systems verified that robot-assisted rehabilitation results in improved performance of functional tasks.

The promising results of robot-assisted rehabilitation systems indicate that robots could be used as effective rehabilitation tools. Different strategies for robot-assisted rehabilitation therapies have been developed, including passive [14,15] active-assistance[8,9,14-18], active-constrained [15], counterpoise control [19] resistive[18], erroramplifying [20-23] , and bimanual modes [15,24,25]. In spite of all these notable studies, questions remain on how best to retrain movement following stroke. Should movement be assisted, assisted only as needed, resisted, manipulated such that movement error is augmented, etc.? Do these training strategies need to be applied separately, or are they more effective when integrated in a specific manner? How do we understand the kinematic and dynamic adaptation of upper-limb movement? In order to investigate such questions a versatile robotic system is needed which can deliver different training therapies thus allow researchers and therapists to evaluate and compare various training strategies implemented with the same platform.

In this work, we develop a versatile robotic system integrated with multiple training modes, i.e., low impedance, passive, assist-as-needed, resist-as-needed, visual error augmentation, viscous force field and force perturbation, to provide a test-bed for the evaluation of these training strategies in upper-limb rehabilitation and the understanding of the motor adaptation in upper-limb movement. Furthermore, the developed robotic system, which is called the PRSAR (Puma Robotic System for Arm Rehabilitation), can combine some of these training modes together so that one can investigate the effect of administering multiple modes simultaneously. The PRSAR is developed based on a PUMA robot and augmented with hand attachment device. An intelligent control framework is designed with a high-level supervisory controller to coordinate different training modes and monitor the task execution and safety, a middle level task planner to generate the reference tasks, and a low-level assistive controller to provide the robotic assistance/resistance in an accurate manner.

This paper is organized as follows. It first introduces the available training modes integrated in the robotic system in Section II. Then Section III presents the development of the robotic system. Section IV shows the results of the validating experiments on unimpaired participants with different training modes. Section V discusses the potential contributions of this work and Section VI concludes the paper and proposes future research directions.

\section{Rehabilitation Training Strategies in PRSAR}

In this section, we will introduce the training task designed for

*Corresponding author: Furui Wang, Abbott Point of Care, Abbott Laboratories, Princeton, NJ, 08540, USA, E-mail: furui.wang@gmail.com

Received October 08, 2013; Accepted November 19, 2013; Published November 21,2013

Citation: Wang F, Barkana DE, Sarkar N (2013) Development of a Versatile Robotic System with Multiple Training Modes for Upper-Limb Rehabilitation Study. Adv Robot Autom 2: 112. doi: 10.4172/2168-9695.1000112

Copyright: () 2013 Wang F, et al. This is an open-access article distributed under the terms of the Creative Commons Attribution License, which permits unrestricted use, distribution, and reproduction in any medium, provided the original author and source are credited. 
the upper-limb rehabilitation, the background and implementation of rehabilitation training strategies integrated in the PRSAR.

\section{The training task}

The training task designed in our work is a reaching task of the arm movement involving cognitive processing that could contribute to a variety of functional daily living activities. The reaching task is commonly used for rehabilitation training of upper extremity after stroke. In this task, the participant is asked to move his/her arm in the forward direction to reach a desired point in the horizontal plane and then bring it back to the starting position repetitively. The reaching task requires a combination of shoulder and elbow motion which could increase the active range of motion (AROM) in the shoulder and the elbow in preparation for later functional reaching activities in rehabilitation. The allowable motion is either restricted in one direction only or in both directions of the 2D horizontal plane, depending on the implementation of specific training modes. The participant is required to follow the tip of a certain position trajectory so that the speed of the arm motion is dictated. The idea here is to improve the ability of the participant's arm movement by helping him/her complete a daily living task at a desirable speed. Improving the speed of movement for such tasks is an important criterion to measure the success of a therapy [26]. Note that the PRSAR system is not limited to the reaching task alone it can implement other rehabilitation tasks involving arm movements. However, in order to describe the training modes we need to choose a task, and in this paper we have chosen the reaching task.

Passive training mode: The passive training mode refers to the training where the user is passive as the robot takes over and moves his/her arm towards a target following a predefined position profile. In passive training mode of the PRSAR, the predefined position profile is a smooth trajectory in $\mathrm{X}$ direction, i.e., moving the arm forward, with a bell-shaped velocity profile (Figure 1).

Low impedance training mode: In the low impedance training mode, the participant actively moves his/her arm while the robot passively follows the arm motion where the impedance of the robot is minimized. In this training mode, the inertia and friction of the robot is compensated by the motor torques of the robot, the participant moves his/her arm in a low impedance environment.

At the beginning of a rehabilitation training session, the participant will participate in the low impedance training mode to learn the manipulation of the robot. The baseline and after-training performance of a participant are also measured during this training mode. Moreover, the participant may need to take extra low impedance training following some deceptive training sessions, e.g., visual error augmentation and viscous force field, to washout any sensory-motor distortion.

Assist-as-needed (ANN) training mode: The assist-as-needed training mode defined in this work is similar to the active assistance strategy in literatures $[8,9,14-18]$. Active assistance is the primary therapy strategy tested so far, where the patient attempts a movement (active) and in which a therapist manually helps complete the movement if the patient is unable to move (assistance) [27]. It is intuitive that in comparison to passive training mode, where the robotic system provides continuous assistance without considering the patient's actual performance, the performance-based active assistance training mode will be more effective. Assisting every movement of a patient has been shown to be not beneficial compared to no assistance or assistance as needed [27]. It has also been suggested $[8,15]$ that performance-based therapy showed better results in improving patients' impairment scores than conventional therapies. Thus, a robot-assisted rehabilitation system could be more efficient if the assistance provided to the patient is given only as and when needed.

In the assist-as-needed training mode of the PRSAR, the participant is required to move his/her arm following the tip of a desired position trajectory as shown in Figure 1. The arm motion is restricted in $\mathrm{X}$ direction only. By tracking the tip of the desired position trajectory, the participant will be actually moving their arm with a predefined velocity profile. The idea here is to improve the motor ability of participant's arm by helping him/her to improve his/her speed of movement, which is an important criterion to measure the success of a therapy.To make the robot assists only as needed, an acceptable position band (Figure 2 ) is defined with the upper bound $x_{\text {upper }}$ and the lower bound $x_{\text {lower }}$ calculated by Equation (1).

$$
\begin{aligned}
& \mathrm{X}_{\text {upper }}=\mathrm{x}_{\mathrm{d}}+\left(\mathrm{x}_{\mathrm{d}}^{*} \text { percentage }\right) \\
& \mathrm{X}_{\text {lower }}=\mathrm{x}_{\mathrm{d}-\mathrm{x}_{\mathrm{d}}}\left(\mathrm{x}^{*} \text { percentage }\right)
\end{aligned}
$$

Where percentage is the value chosen to set the upper and lower bounds for the defined position trajectory. If the actual position $\mathrm{x}(\mathrm{t})$ lies within the acceptable band, then the participant is considered to be able to track the trajectory without robotic assistance. If the actual position $\mathrm{x}(\mathrm{t})$ is not between the upper bound $\mathrm{x}_{\text {upper }}$ and the lower bound $\mathrm{x}_{\text {lower }}$, then the robot is activated to provide assistance to bring the arm position back into the acceptable range.

Note that each participant requires a certain amount of time (settling time) to generate the desired motion. The robot should not be
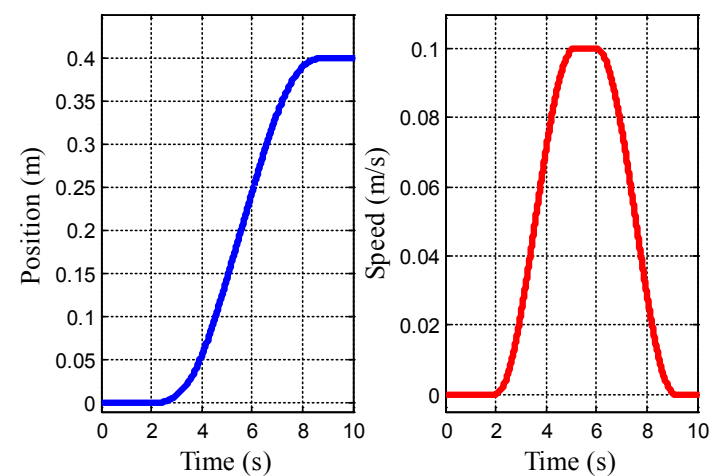

Figure 1: Position and velocity profiles. Here, the distance of the task is $0.4 \mathrm{~m}$ and the maximum speed is $0.1 \mathrm{~m} / \mathrm{s}$.

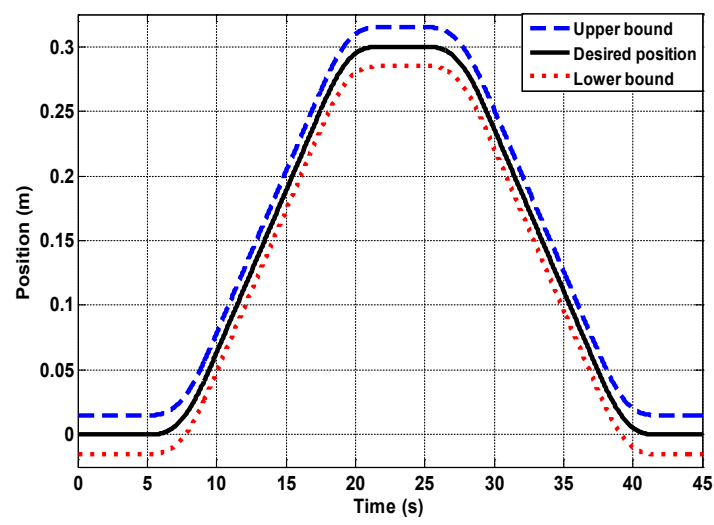

Figure 2: The Acceptable Position Band. 
activated until it is determined that the participant is not able to generate the required motion by his/her own effort. Thus, in our application, the average position is used to determine the activation of the robotic assistance instead of the instant position. During a given time interval, the averages of the actual arm position, $\mathrm{x}_{\text {ave }}$, the upper bound, $\mathrm{x}_{\text {upper_ave' }}$ and the lower bound, $x_{\text {lower_ave }}$, are calculated by Equation (2):

$$
\begin{gathered}
x_{\text {ave }}=\frac{t_{s}}{\left(t_{f}-t_{i}\right)} \cdot \sum_{t_{i}}^{t_{f}} x(t), \quad x_{\text {lower_ave }}=\frac{t_{s}}{\left(t_{f}-t_{i}\right)} \cdot \sum_{t_{i}}^{t_{f}} x_{\text {lower }}(t) \\
x_{\text {upper_ave }}=\frac{t_{s}}{\left(t_{f}-t_{i}\right)} \cdot \sum_{t_{i}}^{t_{f}} x_{\text {upper }}(t)
\end{gathered}
$$

Where $t_{i}$ and $t_{f}$ are the starting time and final time of a time interval, respectively; $t_{s}$ is the sampling time of the data acquisition; $x(t)$ is the actual arm position at any given time $t$.

For any time interval, if condition: $\mathrm{x}_{\text {lower_ave }}<\mathrm{x}_{\text {ave }}<\mathrm{x}_{\text {upper_ave }}$ is satisfied, the assistive controller is not activated and the participant continues the tracking task without robotic assistance. If condition is not satisfied, the robot is activated to provide assistance.

Resist-as-needed (RAN) training mode: Stroke patients may have excessive co-activation or increased stiffness in their affected limbs, so it becomes difficult for them to move their arm in an accurate manner. In the resist-as-needed training mode, the participant is required to move his/her arm on his/her effort from an initial position $\mathrm{O}$ to a target position $\mathrm{T}$ in a straight line following a position profile while his/her arm is able to move in the $2 \mathrm{D}$ horizontal plane. The robot provides resistance to the participant's arm movement if the arm motion deviates from the straight line path. The purpose of this training is to improve the motor ability of the patient's arm move in an accurate manner. The idea of resist-as-needed training comes from the virtual channel training strategies $[28,29]$.

A virtual channel is defined as shown in Figure 3. No robotic resistance will be generated if the arm position remains inside the virtual channel. However, if the arm position goes out of the channel, a spring-like restoring force will be applied to the arm to enforce it back to the right path. The restoring force is,

$$
\mathrm{F}=\left\{\begin{array}{c}
0, \quad|\mathrm{y}(\mathrm{t})| \leq \frac{\mathrm{W}}{2} \\
-\mathrm{K} \cdot\left(\mathrm{y}(\mathrm{t})+\frac{\mathrm{W}}{2}\right), \mathrm{y}(\mathrm{t})<-\frac{\mathrm{W}}{2} \\
-\mathrm{K} \cdot\left(\mathrm{y}(\mathrm{t})-\frac{\mathrm{W}}{2}\right), \quad \mathrm{y}(\mathrm{t})>\frac{\mathrm{W}}{2}
\end{array}\right.
$$

Where $W$ is the width of the virtual channel in $\mathrm{mm}$ and $K$ is the stiffness of the spring-link force field in $\mathrm{N} / \mathrm{mm}$. $W$ and $K$ are adjustable to meet participant's actual motor condition.

Visual error augmentation (VEA) training mode: The latest research in many models and artificial learning systems such as neural networks suggest that error drives sensorimotor learning, so that one can learn adaptation more quickly if the error is larger [30]. Such error-driven learning processes are believed to be central to adaptation and the acquisition of skill in human movement [31,32]. Visual error augmentation can improve the rate and extent of motor learning in healthy participants and may facilitate neuro-rehabilitation strategies that restore function in brain injuries such as stroke [21]. Feedback distortion is shown to be able to elicit functional improvements in patients with chronic stroke and traumatic brain injury [23].
In the VEA training mode, the task is a $1 \mathrm{D}$ reaching task, which is the same with the AAN training mode. However, the arm position trajectory displayed to the participant is amplified by a certain gain factor $K$ so that makes the position error more noticeable to the participant and stimulate him/her to make faster response to correct the position error Figure 4.

The position error

$$
e=x_{d}(t)-x(t)
$$

where, $x(t)$ is the actual arm position, $\mathrm{X}_{\mathrm{d}}(\mathrm{t})$ is the desired arm position.

The arm position displayed to the participant on the monitor, $\mathrm{x}^{\star}$, is

$$
x^{*}=x_{d}(t)+K \cdot\left(x(t)-x_{d}(t)\right)
$$

Viscous force field training (VFF) mode: This training mode is designed to help the understanding of the motor adaptation and motor learning process of the upper-limb during the reaching movement. Stroke patients may have excessive co activation or increased stiffness in their affected limbs. These conditions need to be considered in understanding the stroke rehabilitation. An undesirable environment is created for healthy participants by applying a force field to their arms during the upper-limb movement to simulate these conditions. Examining the adaptation process and after-adaptation effect of the arm movement in the dynamic environment will help understand the kinematic and dynamic influence in motor learning during the goaldirected arm movement as an alternative to study directly with the more vulnerable stroke patients [ 28,33].

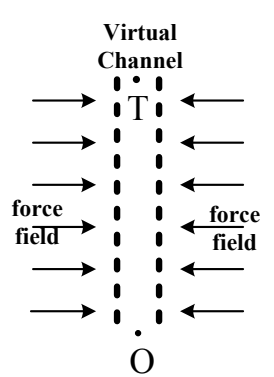

Figure 3: Illustration of the Resist-As-Needed Training.

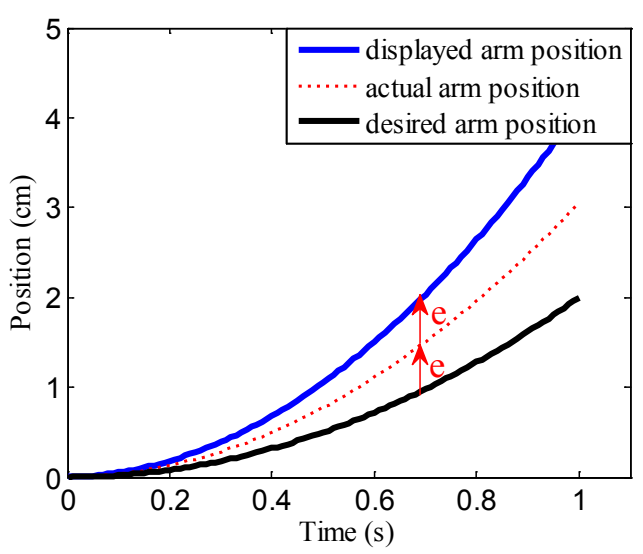

Figure 4: Illustration of the visual error augmentation. The gain of augmentation, $K=2$ in this figure. The red line represents the actual arm position, not displayed to the participant. The blue line and the black line posiont the desired and augmented arm position trajectories. Thes lines are displayed to the participant. 
In the viscous force field training mode, the participant is required to do the reaching task in the $2 \mathrm{D}$ horizontal plane following a position profile along a straight line path in $\mathrm{X}$ direction. A dynamic environment is created so that a viscous force, which is proportional to the arm movement, will be applied perpendicular to the direction of the arm movement (Figure 5). The viscous force is defined as,

$$
\left[\begin{array}{l}
F_{x} \\
F_{y}
\end{array}\right]=\left[\begin{array}{cc}
0 & 0 \\
K_{v} & 0
\end{array}\right] \cdot\left[\begin{array}{l}
v_{x} \\
v_{y}
\end{array}\right]=\left[\begin{array}{c}
0 \\
K_{v} \cdot v_{x}
\end{array}\right]
$$

Force perturbation (FB) training mode: This training mode, similar to the viscous force field training mode, is designed to examine how the upper-limb movements are modified during adaptation to external force perturbations applied on the arm during the reaching task. Instead of creating a dynamic force field, force perturbation is randomly applied to the opposite direction as resistance against the arm movement to simulate the excessive stiffness in the affected arm of stroke patients. The arm motion is restricted in one direction only in the force perturbation training mode.

\section{The Robotic System Development}

\section{Hardware}

A PUMA 560 robotic manipulator is used as the main hardware platform in this work. The PUMA robot is augmented with a forcetorque sensor and a hand attachment device (Figure 6).

The PUMA 560 robot is a 6 degrees-of-freedom (DOF) device consisting of six revolute axes. Each major axis (joints 1, 2 and 3) is equipped with an electromagnetic brake, which can be activated when power is removed from the motors, thereby locking the robot arm in a fixed position. The technical specifications of this robot can be found in [34]. In order to record the force and torque applied by the robot, an ATI Gamma force/torque sensor is used. The robot has been interfaced with Matlab Simulink/Realtime Workshop to allow fast and easy system development. The force values recorded from the force/torque sensor are obtained using a National Instruments PCI-6031E ADC board. The joint angles of the robot are measured by encoders and received by the computer through a Measurement Computing PCIQUAD04 encoder board. The torque output calculated by the Simulink model is sent to the robot through a Measurement Computing PCIM-DDA06/16 DAC board. The sampling rate of all boards is set as $1 \mathrm{kHz}$. A computer monitor is placed in front of the participant to provide visual feedback about his/her motion trajectory during the execution of the task.

In order to provide robotic assistance to the participant's upper arm, a hand attachment device (Figure 6 bottom) is designed to couple the arm to the robot. The hand attachment device consists of an aluminum plate with two small flat-faced electromagnets (Magnetool Inc.), and a steel forearm padded splint (Moore Medical Inc.), which is attached to the aluminum plate by the magnetic power. The participant's arm is strapped on the splint. An ATI Gamma force/torque sensor is placed between the robot and hand attachment device for force measurement. A handheld controller, which controls the power to magnetize and demagnetize the electromagnets, is given to the participant to remove the hand attachment device from the robot in a safe and quick manner if needed.

\section{Control system}

The control architecture of the PUMA robotic system consists of a high-level supervisory controller to coordinate and switch between different training modes, and monitor the task execution and the participant's safety; a middle level task planner to modify task parameters and generate task trajectories; and a low-level assistive controller to execute the task accurately (Figure 7). A graphic user interface (GUI) is developed to provide easy mode selection and task parameter setting. The control system is developed in Matlab/Simulink Real-time Windows Target to interface with the hardware and execute the control program in real-time.

The high-level supervisory controller: The high-level supervisory

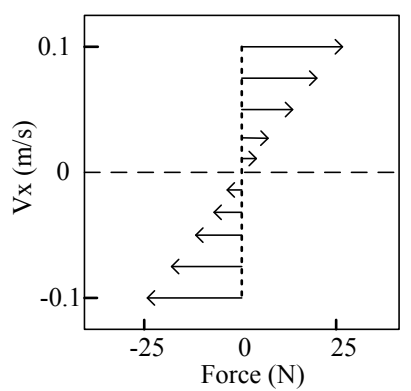

Figure 5: Illustration of Viscous Force Field The viscosity of the force field is $250 \mathrm{~N} /(\mathrm{m} / \mathrm{s})$.

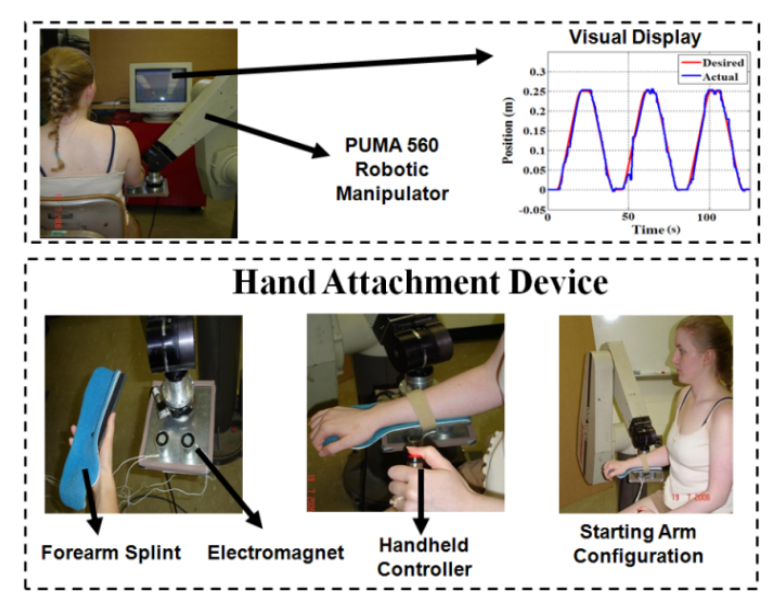

Figure 6: The PUMA Robotic System.

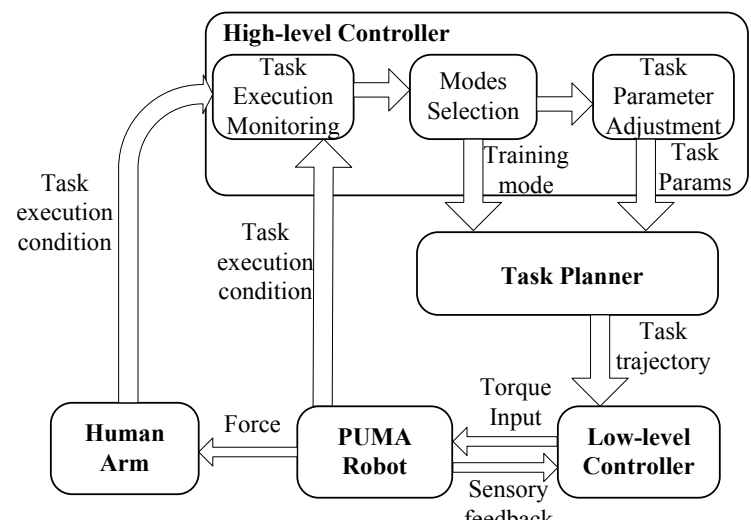

Figure 7: Control Architecture. 
controller is a discrete event system that can be modeled as a deterministic finite automaton. We use a state chart to represent the high-level controller as shown in Figure 8. From the statechart, a vector $S$ is defined to make the training mode selection by setting each bit of the vector to 1 or 0 to enable or disable the corresponding training mode. Each training mode is represented by a sub-state. The AAN, RAN and VEA training modes are designed as parallel sub-states, which means these modes can be enabled alone or in different combinations and work as integrated training modes. Others are exclusive sub-states that cannot be enabled together. The structure of the high-level controller allows flexible and versatile training modes selection of the robotic system.

The middle-level task planner: The task planner receives the mode selection and task parameters from high-level controller and generates the reference task trajectory for the low-level controller to execute. The task planner is a trajectory generator built with Matlab/Simulink $\mathrm{S}$-function. Once a training mode is selected, the total task distance, maximum speed and acceleration, and the DOF of the motion will be defined in the high-level controller. The task planner will use these parameters to generate the reference task trajectory for the low-level controller. The reference trajectory is a smooth position profile with bell-shape velocity profile as shown in Figure 8 .

Graphic user interface: To provide a user-friendly interface which allows mode selection without accessing the Simulink program, a user interface is designed with Matlab GUI to make quick training mode selections and task parameter settings for the experimenter (Figure 9). The left half of the GUI is the mode selection panel. The default button

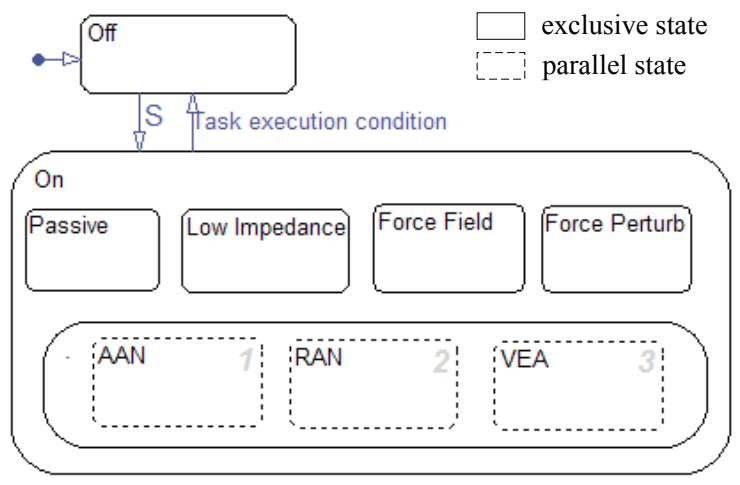

Figure 8: State chart of High-level Supervisory Controller.

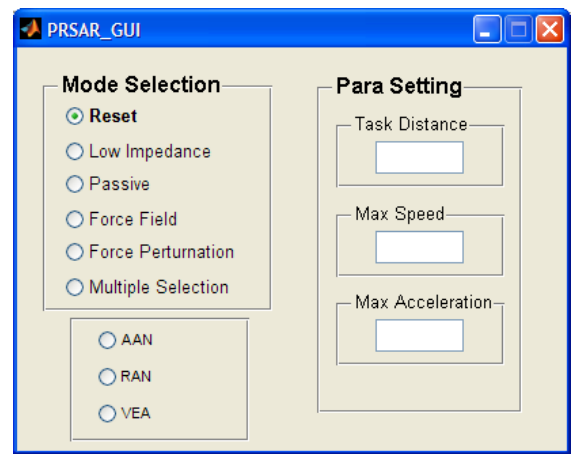

Figure 9: User interface for task selection and parameter setting in GUI. is Reset, which corresponds to the Off state in the state chart. Each training mode has an exclusive selection button, while AAN, RAN and VEA training modes are grouped in a box where multiple selection is permitted. Each button represents a bit in the $S$ vector. Selecting a button will set the corresponding bit to 1 and unselecting a button will set that bit to 0 . The right half of the GUI is the task parameter setting panel. The experimenter can define the total task distance, maximum speed and acceleration by type in the values in text boxes.

The low-level assistive controller: The low-level controller is designed to provide robotic assistive/resistive force to the participant to complete the movement tracking task in the task-space in an accurate manner as required in different training modes. In the PRSAR, the low-level controller is an assistive controller which has an outer force feedback loop and an inner position feedback loop (Figure 10). The tracking of the reference trajectory in $\mathrm{X}$ direction is guaranteed by the inner motion control. The design of the low-level controller has been reported in [35]. The contact force in $\mathrm{X}$ direction, which is given as a force reference to the controller, is computed by a planner. The proposed controller is similar to an impedance controller; however it allows specifying the reference time-varying force directly. A brief discussion of the controller is presented below. by

The equations of the motion in $\mathrm{X}$ direction for the robot are given

$$
\Gamma=u-J^{T}(q) \cdot F=M(q) \ddot{q}+V(q, \dot{q})+G(q)
$$

where $\mathrm{M}(\mathrm{q})$ represents the inertia matrix, $V(\mathrm{q}, \dot{\mathrm{q}})$ is the summation of the Coriolis torques and centrifugal torques, and $\mathrm{G}(\mathrm{q})$ is the vector of gravity torques. $\Gamma$ is the generalized joint force which is calculated using $\mathrm{u}-\mathrm{J}^{\mathrm{T}}(\mathrm{q}) \mathrm{F}$, where $\mathrm{u}$ is the input to the robot, $\mathrm{J}(\mathrm{q})$ is the Jacobian matrix and $\mathrm{F}$ is the contact force exerted by the robot.

Using inverse dynamics control, the robot dynamics are linearized and decoupled via feedback. Control output $u$ to the PUMA robot is designed as follows:

$$
\mathrm{u}=\mathrm{M}(\mathrm{q}) \mathrm{y}+\mathrm{V}(\mathrm{q}, \dot{\mathrm{q}})+\mathrm{G}(\mathrm{q})+\mathrm{J}^{\mathrm{T}} \mathrm{F}
$$

Here, $y=\ddot{\mathrm{q}}$ represents a new input. The new control input $\mathrm{y}$ is designed so as to allow tracking of the desired force $\mathrm{F}_{\mathrm{d}}$. For this purpose, the control law is selected as follows:

$$
\mathrm{y}=\mathrm{J}(\mathrm{q})^{-1} \mathrm{M}_{\mathrm{d}}^{-1}\left(-\mathrm{K}_{\mathrm{d}} \dot{\mathrm{x}}+\mathrm{K}_{\mathrm{p}}\left(\mathrm{x}_{\mathrm{f}}-\mathrm{x}\right)-\mathrm{M}_{\mathrm{d}} \dot{\mathrm{J}}(\mathrm{q}, \dot{\mathrm{q}}) \dot{\mathrm{q}}\right)
$$

where $X_{f}$ is a suitable reference to be related to force error; $M_{d}$ (mass), $\mathrm{K}_{\mathrm{d}}$ (damping) and $\mathrm{K}_{\mathrm{p}}$ (stiffness) matrices specify the target impedance

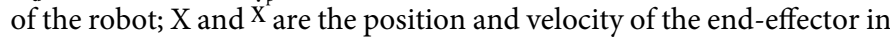
the Cartesian coordinates, respectively. The relationship between the joint space and the Cartesian space acceleration is used to determine position control equation:

$$
\ddot{x}=J(q) \ddot{q}+\dot{J}(q, \dot{q}) \dot{q}=J(q) y+\dot{J}(q, \dot{q}) \dot{q}
$$

By substituting Equation (9) into Equation (10),

$$
\begin{aligned}
& \ddot{\mathrm{x}}=\mathrm{J}(\mathrm{q})\left\{\mathrm { J } ( \mathrm { q } ) ^ { - 1 } \mathrm { M } _ { \mathrm { d } } ^ { - 1 } \left[-\mathrm{K}_{\mathrm{d}} \dot{\mathrm{x}}+\mathrm{K}_{\mathrm{p}}\left(\mathrm{x}_{\mathrm{f}}-\mathrm{x}\right)-\right.\right. \\
& \left.\left.\quad \mathrm{M}_{\mathrm{d}} \mathrm{J}(\mathrm{q}, \dot{\mathrm{q}}) \dot{\mathrm{q}}\right]\right\}+\dot{\mathrm{J}}(\mathrm{q}, \dot{\mathrm{q}}) \dot{\mathrm{q}} \\
& =-\mathrm{M}_{\mathrm{d}}^{-1} \mathrm{~K}_{\mathrm{d}} \dot{\mathrm{x}}+\mathrm{M}_{\mathrm{d}}{ }^{-1} \mathrm{~K}_{\mathrm{p}}\left(\mathrm{x}_{\mathrm{f}}-\mathrm{x}\right) \\
& \text { Thus, } \\
& M_{d} \ddot{x}+K_{d} \dot{x}+K_{p} x=K_{p} x_{f}
\end{aligned}
$$

Equation (12) shows the position control tracking of $x$ with 


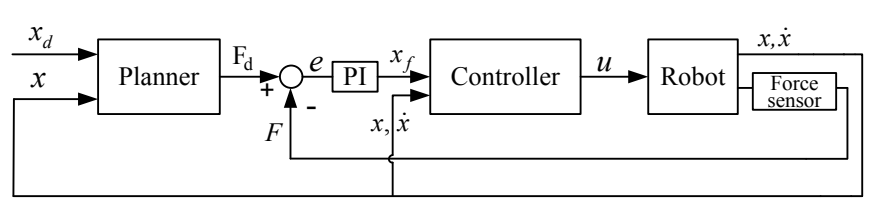

Figure 10: The Assistive controller.

dynamics specified by the choices of $\mathrm{K}_{\mathrm{d}}, \mathrm{K}_{\mathrm{p}}$ and $\mathrm{M}_{\mathrm{d}}$ matrices. Impedance is attributed to a mechanical system characterized by these matrices that allows specifying the dynamic behavior. Let Fd be the desired force reference, which is computed using a PID loop:

$$
\mathrm{F}_{\mathrm{d}}=\mathrm{P}_{\mathrm{d}}\left(\mathrm{x}_{\mathrm{d}}-\mathrm{x}\right)+\mathrm{I}_{\mathrm{d}} \int\left(\mathrm{x}_{\mathrm{d}}-\mathrm{x}\right) \mathrm{dt}+\mathrm{D}_{\mathrm{d}} \frac{\mathrm{d}\left(\mathrm{x}_{\mathrm{d}}-\mathrm{x}\right)}{\mathrm{dt}}
$$

Where $\mathrm{X}_{\mathrm{d}}, x, \mathrm{P}_{\mathrm{d}}, \mathrm{I}_{\mathrm{d}}$ and $\mathrm{D}_{\mathrm{d}}$ are the desired position, actual position, the proportional, integral and derivative gains of the PID position loop, respectively. The relationship between $\mathrm{X}_{\mathrm{f}}$ and the force error is expressed in Equation (14) as:

$$
\mathrm{x}_{\mathrm{f}}=\mathrm{P}\left(\mathrm{F}_{\mathrm{d}}-\mathrm{F}_{\mathrm{i}}\right)+\mathrm{I} \int\left(\mathrm{F}_{\mathrm{d}}-\mathrm{F}_{\mathrm{i}}\right) \mathrm{dt}
$$

where $P$ and $I$ are the proportional and integral gains, respectively. The $P$ and $I$ gains are tested in our previous work to guarantee a smooth and sufficient assistance to the participant [36]. Fi is the force applied by the human. Equations (12) and (14) are combined to obtain below equation:

$$
\mathrm{M}_{\mathrm{d}} \ddot{\mathrm{x}}+\mathrm{K}_{\mathrm{d}} \dot{\mathrm{x}}+\mathrm{K}_{\mathrm{p}} \mathrm{x}=\mathrm{K}_{\mathrm{p}}\left(\mathrm{P}\left(\mathrm{F}_{\mathrm{d}}-\mathrm{F}_{\mathrm{i}}\right)+\mathrm{I} \int\left(\mathrm{F}_{\mathrm{d}}-\mathrm{F}_{\mathrm{i}}\right) \mathrm{dt}\right)
$$

From Equation (15), the desired force response is achieved by controlling the position of the robot.

Besides the assistive/resistive force in $\mathrm{X}$ direction, the low-level controller also provides viscous force and resistive force in $\mathrm{y}$ direction in RAN and VFF training modes. In this case, an extra term ${ }^{\mathrm{y}}$ in the contact force tern F is calculated using Equation (3) and (6) and then plugged into Equation (8) to compute the control output to the PUMA robot.

Safety consideration: Ensuring safety of the participant is a very important consideration when designing a robot-assisted rehabilitation system. Thus, in case of emergency situations, the experimenter can press a kill switching button to stop the PUMA robot. With the quickrelease hand attachment device, the participant can also quickly release his/her arm from the PUMA robot by pressing the handheld controller to deal with any physical safety related events. When the controller is pressed, the electromagnets are demagnetized instantaneously and the participant is free to remove the splint from the robot.

Moreover, rotation angle and torque limits of each joint of the robot are monitored within the control system to disable and stop the robot with its inherited brakes to prevent unexpected movement or excessive motor torque.

\section{Validating Experiments and Results}

In this section we present the validating experiments conducted on unimpaired participants to illustrate the implementation of different training modes with the proposed robotic system. Note that the experiments focus on the validation of the developed robotic system to administer different training modes. As a result, we used unimpaired participants to demonstrate the functionality of the robotic system, PRSAR. In the future, we will systematically study the impact of different training strategies on stroke rehabilitation using PRSAR.

\section{Procedure}

Three participants with no arm impairement were recruited for the experiments. One participant participated in the experiments with Low-Impedance, AAN, RAN and VEA training modes. The second participant participated in the experiments with viscous force field and force perturbation training modes. The third participant participated in the integrated training of AAN and VEA modes. The total distance and the maximum speed of the reaching movement were set as $0.25 \mathrm{~m}$ and $0.05 \mathrm{~m} / \mathrm{s}$, respectively. The participants were required to repeat the forward and backward reaching task 5 times in each training mode.

In the AAN training mode, the time interval to calculate the average position and determine the activation of the robotic assistance was chosen to be 4 second. In the RAN training mode, the width of the virtual channel was set as $0.01 \mathrm{~m}$, which was $5 \mathrm{~mm}$ deviation from the desired path on each side. The stiffness of the restoring force was $2500 \mathrm{~N} / \mathrm{m}$., which was tested to be sufficient to bring the arm back into the channel quickly. In the VEA training mode, the gain of the visual error augmentation was chosen as 2 , which had been shown to elicit the best training performance in literature [21]. The augmented and desired arm position trajectories were displayed to the participant on monitor screen. In the viscous force field training mode, the viscosity of the force field was $500 \mathrm{~N} /(\mathrm{m} / \mathrm{s})$. In the force perturbation training mode, the amplitude of the perturbation force was $20 \mathrm{~N}$ and the duration of the force perturbation was 1 second, applied at $t=12,32,52$, 72 and 92 seconds. In the integrated training, the participant received the augmented arm position trajectories displayed on the monitor while the robotic assistance was available to assist the participant when needed.

Participants were seated in a height adjustable chair as shown in Figure 6 (top left) and were required to place their forearm on the hand attachment device as shown in Figure 6 (bottom left). The height of the endpoint of the PUMA robot was adjusted for each participant to start the tracking task in the same arm configuration. The starting arm configuration was selected as shoulder at neutral $0^{\circ}$ position and elbow at $90^{\circ}$ flexion position. The task required moving the arm in forward flexion to approximately $60^{\circ}$ in conjunction with elbow extension to approximately $0^{\circ}$ and then coming back to the starting position. The release button of the hand attachment device was given to the participants in case of emergency situations during the task execution (Fig. 6- bottom middle). The participants received visual feedback of the task trajectories and their own position trajectories on a computer monitor in front of them (Figure 6-top right). The actual arm position and contact force were recorded at $1 \mathrm{kHz}$ sampling rate.

\section{Experimental results}

The first participant participated in the Low Impedance, AAN, RAN and VEA training modes. In all these training modes, the participant was asked to follow a reference trajectory in $\mathrm{X}$ direction displayed on the monitor screen. Even though the participant had no arm impairment, it was not easy to perfectly track the desired trajectory. By comparing the actual arm position trajectory with the desired trajectory, we can evaluate the efficacy of each training mode. The position trajectories in the task direction (X direction) during all training modes are shown in Figure 11. The averages of the absolute position errors in different training modes are: $e_{\mathrm{LI}}=5.3 \mathrm{~mm}, \mathrm{e}_{\mathrm{AAN}}=4.5 \mathrm{~mm}, \mathrm{e}_{\mathrm{RAN}}=5.2 \mathrm{~mm}$ and $\mathrm{e}_{\mathrm{VEA}}=3.9 \mathrm{~mm}$. Note that the low-impedance mode can be considered as the baseline performance for the participant. In the Low Impedance and RAN training modes, the participant was able to move their arm in 
Y direction also. By comparing the deviation from the task direction, we can examine the improvement of the participant's ability to conduct the training task in the right direction. The position trajectories of the participant in X-Y plane in Low Impedance and RAN training modes are shown in Figure 12. The averages of absolute position errors in $\mathrm{Y}$ direction are $3.87 \mathrm{~mm}$ in the Low Impedance training and $2.66 \mathrm{~mm}$ in the RAN training.

The second participant participated in the VFF and FP training modes. The purpose of these experiments was to see how the participant would adapt to dynamic environment during arm movement thus to help understand the kinematic and dynamic influence in motor learning during a goal-directed arm movement. The participant was allowed to move his arm in the X-Y plane in the VFF training mode. The arm position trajectory and the corresponding viscous force applied in the VFF training mode are shown in Figure 13. The viscous force was applied perpendicular to the task direction properly

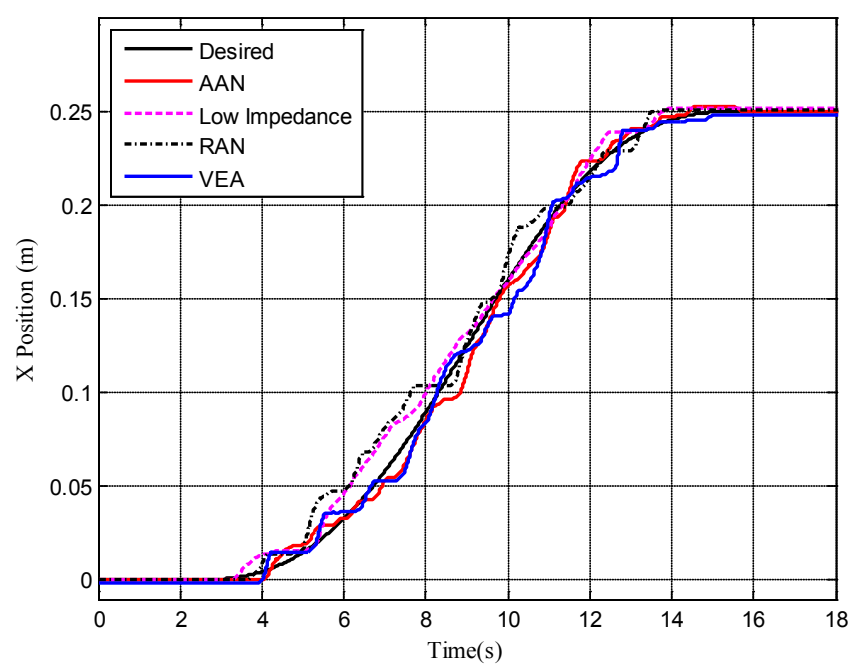

Figure 11: Position Trajectories in Different Training Modes.

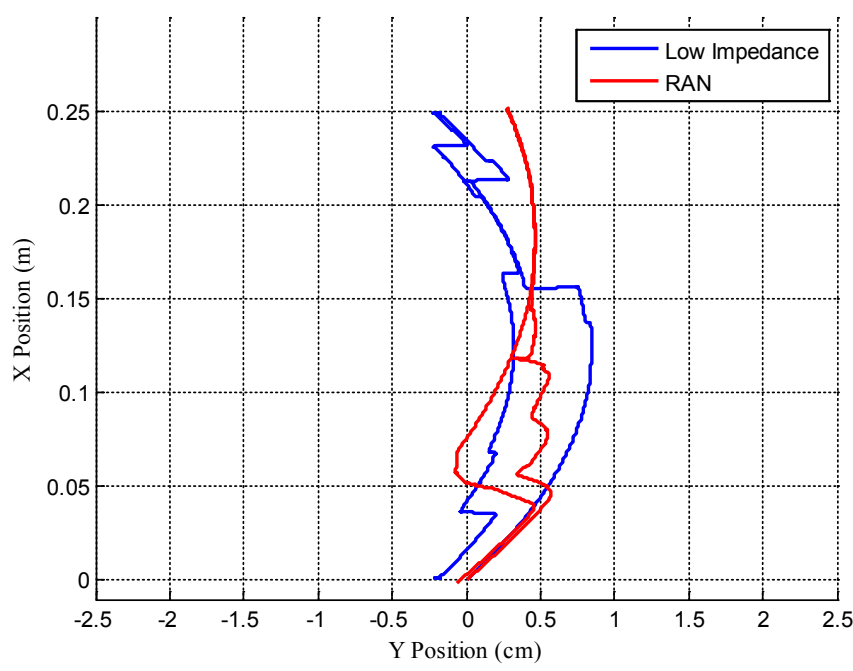

Figure 12: Position trajectories in Low Impedance and RAN trainings. The restoring force keeps the arm from moving out of the virtual channel in the RAN Training mode. as defined in Equation (6) during the arm movement. Although deviation from the task line was observed during the movement, the participant managed to reach the target position under the influence of the force field. Exploring the adaptation process of the arm movement under the influence of the dynamic force field will be an alternative to help understand motor learning process of the affect limbs of stroke patients. In the FP training mode, the participant was allowed to move his arm in X direction only. The position error trajectory and the force perturbation during the FP training mode are shown in Figure 14. The position error increased significantly during the periods when the force perturbation was applied. Errors in opposite direction were observed right after the force perturbation, which could be an indication of adaptation in the motor learning. This training mode can be viewed as a simulation of motor learning process of those stroke patients whose affected limbs have excessive muscle stiffness at certain configuration during arm movement.

The third participant participated in the integrated training of AAN and VEA modes. In our previous work [37], we have shown that by combining some of the training modes, it is possible to improve the efficiency of the rehabilitation training. It is of great interests to see whether more training modes can be integrated to provide optimal training strategies for stroke rehabilitation. The desired, actual and displayed arm position trajectories are shown in Figure 15 up. The deviation from the displayed arm position to the desired arm position was twice as the real position error from actual arm position to the desired arm position. The corresponding position error and the activation of robotic assistant are shown in Figure 15 down. Once activated, the robotic assistance would continue for 4 seconds and then bring the arm position back into the acceptable error band. Note that, the robotic assistance was activated based on average position error not instant position error. For example, at $\mathrm{t}=20.5$, where the instant position error was out of the acceptable error band, the robotic assistance was not activated. It was activated until $t=24 \mathrm{~s}$, when the average position error in $4 \mathrm{~s}$ interval $(\mathrm{t}=20 \sim 24 \mathrm{~s})$ was out of acceptable error band. The average position is computed using Equation (2) in Section 2.4. By comparing the training performance in this training mode with other training modes, we want to find optimal training strategies for stroke patients with different levels and types of arm impairments.

\section{Discussion}

With the large and ever increasing stroke population and the huge amount of stroke related medical cost, robot-assisted rehabilitation has become an active research area during recent years, aiming to deliver reliable and standardized rehabilitation therapies as well as reduce the cost of rehabilitation. Various robotic systems have been developed and different rehabilitation training strategies have been proposed to improve the rehabilitation training efficacy. The goal of our present work is to provide a general test-bed to evaluate and compare different training strategies. A PUMA robotic system for arm rehabilitation (PRSAR) is developed in this paper. The PRSAR is integrated with different training strategies for the study of upper-limb rehabilitation after stroke. A similar system, MIME, has been reported by Lum in 2002 [15], however, the implementation for assist-as-needed and resist-as-needed training strategies proposed in this work are different. Additionally, new training strategies reported in recent literatures, e.g., visual error augmentation, viscous force field and force perturbation modes, have been implemented in the PRSAR to enhance the versatility of the robotic system. Moreover, in our system, some of these training modes can be implemented together as new integrated training 


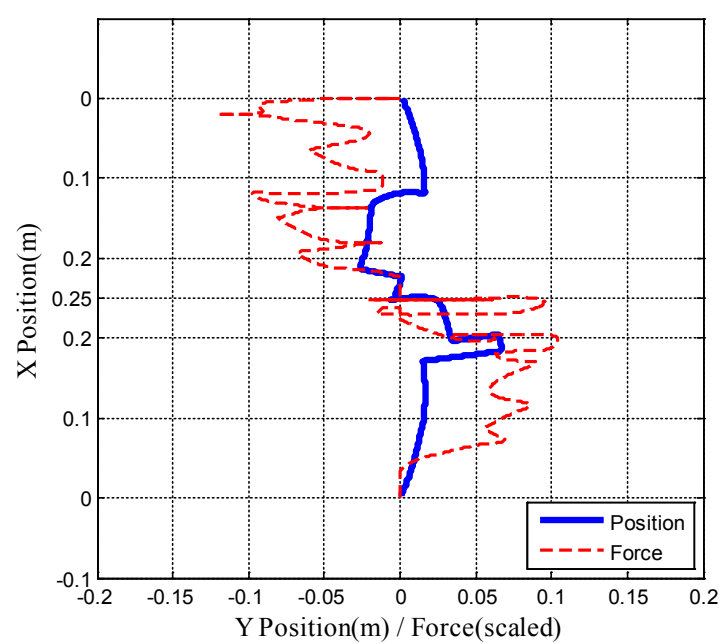

Figure 13: Position and Force Trajectory in Viscous Force Field Training Note the backward movement is flipped around $Y=0.25 \mathrm{~m}$ line for better demonstration.

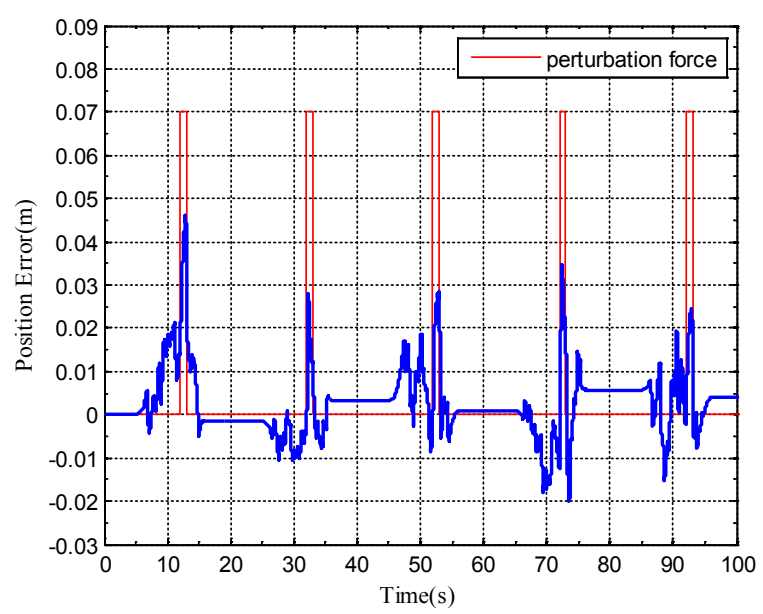

Figure 14: Position and Force Trajectory in Force Perturbation Training. The perturbation force is scaled down for readability.

modes under the coordination of the presented high-level supervisory controller (Figure 15). With all the available training modes, this PRSAR has the potential to serve as a test-bed to evaluate and compare the training efficacy of different training strategies for upper-limb rehabilitation and to understand the motor learning and adaptation process during upper-limb movement. We are aware that PUMA robot was not originally developed for rehabilitation purposes and thus have a few drawbacks in this regard. However, with improved control and planning, PUMA robot has been shown to be useful in rehabilitation purpose as well [15]. The primary purpose of our work is to develop a versatile control architecture that can provide different training modes. To implement this architecture we have used the PUMA robot because of availability. But note that our development is not restricted to PUMA robot alone.

The control system of the robotic system has a hierarchical architecture consisting of a high-level supervisory controller, a middlelevel planner, and a low-level assistive controller. The high-level supervisory controller and middle-level planner, which work together to coordinate different training modes, adjust task parameters, generate task trajectories and monitor the task execution, are flexible and expandable. They can be integrated in other robotic systems to produce the same reference profiles for reaching task with minor modifications. Furthermore, new training strategies, if discovered in future works, can be integrated to the control architecture by adding these training strategies as new sub-states into the state chart of the high-level controller without changing other states in the control architecture.

The enhanced PRSAR was tested with unimpaired participants in the initial validating experiments. A reaching movement was chosen for the training task but the PRSAR is not restricted to this task only. The experimental process proved that all training modes worked properly. The robotic assistive/resistive/viscous/perturbation forces were enabled and applied as designed. The AAN and VEA modes were able to work together as an integrated training mode. The performance of the unimpaired participant showed differences among Low-Impedance, AAN, RAN and VEA training modes (Figures 11,12). The viscous force field and force perturbation showed impact to the motor adaptation during the arm movement in the VFF and FP training modes (Figures 13,14). The efficacy of these training modes in upper-limb rehabilitation and the influence in motor adaptation during arm movement will be further evaluated with formally designed experiments and adequate subject population.

\section{Conclusion and Future Work}

The presented robotic system (PRSAR) is capable of providing multiple training modes to facilitate a test-bed for the study of upperlimb rehabilitation after stroke. The control system designed for this robotic system consists of a flexible and expandable high-level supervisory controller to coordinate different training modes, modify task parameters and monitor the task execution and the participant's safety, a middle level task planner to generate task trajectories, and a low-level assistive controller to execute the task accurately. The presented robotic system has been tested in the initial experiments and all training modes work as designed.

As a future work, experiments will be conducted on both healthy
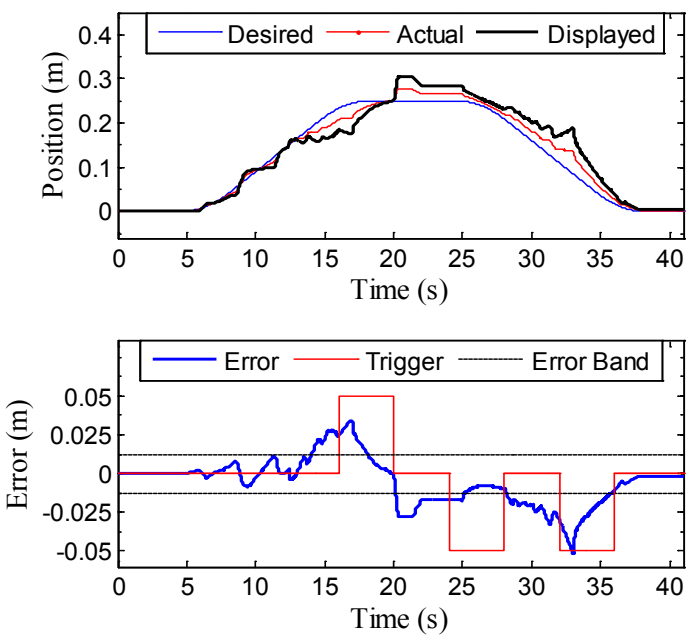

Figure 15: Up: Desired, actual and displayed (error augmented) arm position. Down: Position error (blue line) and triggering of robotic assistance in the integrated training of AAN and VEA modes. 
Citation: Wang F, Barkana DE, Sarkar N (2013) Development of a Versatile Robotic System with Multiple Training Modes for Upper-Limb Rehabilitation Study. Adv Robot Autom 2: 112. doi: 10.4172/2168-9695.1000112

and stroke participants with different training modes to evaluate the efficacy of different training strategies and understand the kinematic and dynamic adaptation in arm movement. Further, the robotic system will be coupled with a hand exoskeleton under development in our research group for the study of the upper-limb reach-to-grasp activity.

\section{References}

1. American Stroke Association "Statistics about Stroke".

2. Matchar DB, Duncan PW (1994) Cost of stroke. Stroke Clin Updates 5: 9-12.

3. Bonita R, Steward A, Beaglehole R (1990) International trends in stroke mortality: 1970-1985. Stroke 21: 989-992.

4. Broderick JP, Phillips SJ, Whisnant JP, O'Fallon WM, Bergstralh EJ (1989) Incidence rates of stroke in the eighties: the end of the decline in stroke? Stroke 20: 577-582.

5. Gray CS, French JM , Bates D, Cartlidge NE, James OF, Venables G (1990) Motor recovery following acute stroke. Age Ageing 19: 179-184.

6. Krebs HI, Palazzolo JJ, Dipietro L, Ferraro M, Krol J, et al. (2003) Rehabilitation Robotics: Performance-Based Progressive Robot-Assisted Therapy. Autonomous Robots $15: 7-20$.

7. Krebs $\mathrm{H}$, Ferraro M, et al. (2004) Rehabilitation robotics: pilot trial of a spatial extension for MIT-Manus. J Neuroeng Rehabil 1: 5.

8. Kahn L, Zygman M, et al. (2006) Robot-assisted reaching exercise promotes arm movement recovery in chronic hemiparetic stroke: a randomized controlled pilot study. J Neuroeng Rehabil 3: 12

9. Leonard EK, Peter SL, Zev WR, David JR (2006) Robot-assisted movement training for the stroke-impaired arm: Does it matter what the robot does? J Rehabil Res Dev 43:619-630.

10. Lum P, Burgar C, et al. (1999) Quantification of force abnormalities during passive and active-assisted upper-limb reaching movements in post-stroke hemiparesis. IEEE Trans Biomed Eng 46: 652-662.

11. Charles GB, Peter SL,Peggy CS,Machiel Van der Loos HF(2000) Development of robots for rehabilitation therapy: the Palo Alto VA/Stanford experience. J Rehabil Res Dev 37: 663-673.

12. Lum $P$, Burgar C, Machiel VL, Peggy CS, Matra M, et al. (2006) MIME robotic device for upper-limb neurorehabilitation in subacute stroke subjects: A followup study. J Rehabil Res Dev 43: 631-642.

13. Loureiro R, Amirabdollahian F, et al.(2003) Upper Limb Robot Mediated Stroke Therapyâ GENTLE/s Approach Autonomous Robots 15: 35-51.

14. Volpe BT, Krebs HI, Hogan N, Edelstein L, Diels C, Aisen M (2000) A nove approach to stroke rehabilitation: robot-aided sensorimotor stimulation. Neurology 54: 1938-1944.

15. Lum PS, Charles GB, Peggy CS, Matra M, Machiel V (2002) Robot-assisted movement training compared with conventional therapy techniques for the rehabilitation of upper-limb motor function after stroke. Arch Phys Med Rehabi 83: 952-959.

16. Hesse S, Schulte-Tigges G, Konrad M, Bardeleben A, Werner C (2003) Robotassisted arm trainer for the passive and active practice of bilateral forearm and wrist movements in hemiparetic subjects. Arch Phys Med Rehabil 84: 915-920.

17. Zygman ML, Rymer WZ, Reinkensmeyer DJ, Kahn LE (2001) Effect of robotassisted and unassisted exercise on functional reaching in chronic hemiparesis. Engineering in Medicine and Biology Society 2: 1344-1347.

18. Susan EF, Hermano IK, Joel S,Walter RF, Neville H (2003) Effects of robotic therapy on motor impairment and recovery in chronic stroke. Arch Phys Med Rehabil 84: 477-482.

19. David JR, Craig DT, Wojciech KT, Andrea NR, Leonard EK (2001) Design of robot assistance for arm movement therapy following stroke. Advanced Robotics 14: 625-637.
20. Bambi RB, Roberta K, Yoky M (2004) Effects of visual feedback distortion for the elderly and the motor-impaired in a robotic rehabilitation environment. Robotics and Automation 2: 2080-2085.

21. Wei Y, Bajaj P, Scheidt R, Patton J (2005) Visual error augmentation for enhancing motor learning and rehabilitative relearning. 9th International Conference on Rehabilitation Robotics.

22. Patton JL, Mussa-Ivaldi FA, Rymer WZ (2001) Altering movement patterns in healthy and brain-injured subjects via custom designed robotic forces in Engineering in Medicine and Biology Society 2: 1356-1359.

23. Brewer B, Klatzky R, Matsuoka Y (2008) Visual feedback distortion in a robotic environment for hand rehabilitation. Brain Res Bull75: 804-813.

24. Lum SP, Lehman SL, Reinkensmeyer DJ (1995)The bimanual lifting rehabilitator: an adaptive machine for therapy of stroke patients. Rehabilitation Engineering 3:166-174.

25. Lum SP, Lehman SL, Reinkensmeyer DJ (1993) Robotic assist devices for bimanual physical therapy: preliminary experiments. Rehabilitation Engineering, IEEE Transactions 1:185-191.

26. Taub E, Uswatte G, Pidikiti R (1999) Constraint-Induced Movement Therapy: A New family of techniques with broad application to physical rehabilitation - a clinical review. J. of Rehab. Research and Development 36: 237-251.

27. David JR, Jeremy LE, Steven CC (2004) Robotics, motor learning, and neurologic recovery. Annu Rev Biomed Eng 6:497-525.

28. Robert AS, David JR, Michael AC, Zev WR, Mussa-Ivaldi FA (2000) Persistence of motor adaptation during constrained, multi-joint, arm movements $\mathrm{J}$ Neurophysiol 84:853-862.

29. Rumelhart DE, Hinton GE, Williams RJ(1986) Learning representations by back-propagating errors. Nature 323: 533-536.

30. M Kawato (1990) Feedback-error-learning neural network for supervised learning. Advanced neural computers

31. Wolpert DM, Ghahramani Z, Jordan MI (1995) An internal model for sensorimotor integration Science 269: 1880-1882.

32. Scharver C, Patton J, Kenyon R, Kersten E (2005) Comparing adaptation of constrained and unconstrained movements in three dimensions. IEEE 9th International Conference on Rehabilitation Robotics.

33. Rand MK, Shimansky Y, Stelmach GE, Bloedel JR (2004) Adaptation of reachto-grasp movement in response to force perturbation Experimental Brain Research 154:50-65.

34. Corke PI, Armstrong-Helouvry B (1994) A search for consensus among mode parameters reported for the PUMA 560 robot. Robotics and Automation 2:1608-1613

35. Sciavicco L, Siciliano B (1996) Modeling and Control of Robot Manipulators

36. Erol D, Sarkar N (2007) Design and Implementation of an Assistive Controller for Rehabilitation Robotic Systems. International Journal of Advanced Robotic Systems 4: 3.

37. Furui W, Barkana ED, Sarkar N (2010) Impact of Visual Error Augmentation When Integrated With Assist-as-Needed Training Method in Robot-Assisted Rehabilitation. IEEE Trans Neural Syst Rehabili Eng 18:571-579. 